\title{
A Window-Based Self-Organizing Feature Map (SOFM) for Vector Filtering Segmentation of Color Medical Imagery
}

\author{
Ioannis M. Stephanakis ${ }^{1,2}$, George C. Anastassopoulos ${ }^{3,4}$, and Lazaros Iliadis ${ }^{4,5}$ \\ ${ }^{1}$ Hellenic Telecommunication Organization S.A. (OTE), \\ 99 Kifissias Avenue, GR-151 24, Athens, Greece \\ stephan@ote.gr \\ ${ }^{2}$ Technological Educational Instutute of Pireaus, \\ GR-122 44, Pireaus, Greece \\ ${ }^{3}$ Democritus University of Thrace, Medical Informatics Laboratory, GR-681 00, \\ Alexandroupolis, Greece \\ anasta@med.duth.gr \\ ${ }^{4}$ Hellenic Open University, Parodos Aristotelous 18, GR-262 22, Patras, Greece \\ ${ }^{5}$ Department of Forestry \& Management of the Environment and Natural Resources, \\ Democritus University of Thrace, GR-68200, Orestiada, Hellas \\ liliadis@fmenr.duth.gr
}

\begin{abstract}
Color image processing systems are used for a variety of purposes including medical imaging. Basic image processing algorithms for enhancement, restoration, segmentation and classification are modified since color is represented as a vector instead of a scalar gray level variable. Color images are regarded as two-dimensional (2-D) vector fields defined on some color space (like for example the RGB space). In bibliography, operators utilizing several distance and similarity measures are adopted in order to quantify the common content of multidimensional color vectors. SelfOrganizing Feature Maps (SOFMs) are extensively used for dimensionality reduction and rendering of inherent data structures. The proposed windowbased SOFM uses as multidimensional inputs color vectors defined upon spatial windows in order to capture the correlation between color vectors in adjacent pixels. A $3 \times 3$ window is used for capturing color components in uniform color space $\left(L^{*} u^{*} v^{*}\right)$. The neuron featuring the smallest distance is activated during training. Neighboring nodes of the SOFM are clustered according to their statistical similarity (using the Mahalanobis distance). Segmentation results suggest that clustered nodes represent populations of pixels in rather compact segments of the images featuring similar texture.
\end{abstract}

Keywords: Vector Filtering, Color Segmentation, Self-Organizing Feature Maps (SOFM), Medical Imaging.

\section{Introduction}

Color image processing systems [1] are used for a variety of purposes, ranging from capturing and rendering scenes in entertainment, industry, scientific exploration and 
medical praxis [2]. Processing of color imagery for feature and edge extraction [3] underlies several image processing algorithms for pattern recognition in industrial and scientific applications, computer vision systems and image coding methods. Color medical images may be enhanced or segmented using such techniques as vector filtering and tensor analysis [4], [5]. Colors are perceived as combinations of the three primary colors, red $(\mathrm{R})$, green $(\mathrm{G})$ and blue $(\mathrm{B})$. The attributes generally used to distinguish one color from another are brightness, hue and saturation. There are several standard color spaces that are widely used in image processing like RGB, CMY, HIS, YIQ and others. All systems can be calculated from the tristimuli R, G, B by appropriate transformations. However, these models are not uniform color spaces [6]. In a uniform color space - such as $L^{*} u^{*} v^{*}$ or $L^{*} a^{*} b^{*}$ [7] - the difference between two colors can be simply measured by their Euclidean distance. In $L^{*} u^{*} v^{*}$ color space, $u^{*}$ and $v^{*}$ represent color chromaticities and $L^{*}$ stands for the intensity.

\subsection{Vector Filtering for Color Images}

Color images are two-dimensional (2-D) vector fields defined on color spaces. Filtering schemes operate on the premise that an image can be subdivided into small regions, each of which can be treated as stationary [8]. Several window shapes and sizes may be used. The most commonly used window is of rectangular shape. Vector filtering is based on the ordering of aggregated distances $D_{i}=\sum_{j=1}^{N} d\left(\mathbf{x}_{i}, \mathbf{x}_{j}\right)$ or aggregated similarities $D_{i}=\sum_{j=1}^{N} s\left(\mathbf{x}_{i}, \mathbf{x}_{j}\right)$ within the sliding window and is nonlinear in nature [9]. The most popular vector filter is the Vector Median Filter (VMF). Non-linear and linear approaches may be combined together in successive processing steps [10]. The proposed approach implements a Self-Organizing Feature Map (SOFM) whose trained neurons may be viewed as the possible outputs of a nonlinear vector operator defined upon a 3x3 rectangular sliding window. The trained neurons capture structural as well as color local information of the underlying segment of the image. Linear statistical analysis in $L^{*} u^{*} v^{*}$ color space is carried out as a second processing step. The number of non-zero eigenvalues of the distributions of the training vectors indicates the dimensionality of the probability distributions of the local patterns that are represented by a neuron of the SOFM.

Traditional image filtering techniques usually applied scalar filters on each channel separately since each individual channel of a color image can be considered as a monochrome image. However, this does not take into account the correlation that exists between the color components of natural images when represented in a correlated color space.

\subsection{Dimensionality Reduction Algorithms and Self-Organizing Feature Maps}

The SOFM [11] is an efficient method for cluster analysis of a high-dimensional feature space onto 2-D arrays of reference vectors. SOFMs are considered as unsupervised learning algorithms and they are frequently used when there exists noapriori knowledge about the distributions of the features (see for example [12]). 
Recent advancements in the subject include Visualization-induced SOM (ViSOM) [13] and other multidimensional scaling (MDS) techniques for structural dimensionality reduction like the ISOMAP (isometric feature mapping) algorithm. Dimensionality reduction is associated as well with principal component analysis (PCA), multilinear principal component analysis (MPCA), linear discriminant analysis (LDA), marginal Fisher analysis and other approaches [14]. Dimensionality reduction should be targeted at removing the dimensions that are unreliable for the classification in order to boost classification accuracy. Retaining small eigenvalues results in overfitting models and complicates calculations. A processing step that involves linear subspace analysis for the local distributions that are represented by a neuron of a SOFM yields a better understanding of the local statistics and improves clustering results.

\section{A Window-Based 2-D SOFM Model for Rendering Color Correlation}

\subsection{Model Formulation}

There are three basic steps involved in the application of the SOFM algorithm after initialization, namely, sampling, similarity matching and updating. These three steps are repeated until map formation is completed. The algorithm is summarized as follows:

1. Initialization. Choose the initial values for the weight vectors $\mathbf{w}_{j}(0)$, which in the proposed approach are comprised by the color vectors within a sliding window of $N \times M$ pixels, i.e. $\mathbf{w}_{j}=\left(\begin{array}{cccc}L_{j, 1}^{*} & L_{j, 2}^{*} & \cdots & L_{j, M x N}^{*} \\ u_{j, 1}^{*} & u_{j, 2}^{*} & \cdots & u_{j, M x N}^{*} \\ v_{j, 1}^{*} & v_{j, 2}^{*} & \cdots & v_{j, M x N}^{*}\end{array}\right)$

where index $j$ equals $1,2, \ldots, L$.

2. Sampling. Choose randomly a window and the corresponding color array

$$
\mathbf{v}(m, n)=\left(\begin{array}{lllll}
L^{*}\left(m-\frac{M}{2}, n-\frac{N}{2}\right) & \cdots & L^{*}(m, n) & \cdots & L^{*}\left(m+\frac{M}{2}, n+\frac{N}{2}\right) \\
u^{*}\left(m-\frac{M}{2}, n-\frac{N}{2}\right) & \cdots & u^{*}(m, n) & \cdots & u^{*}\left(m+\frac{M}{2}, n+\frac{N}{2}\right) \\
v^{*}\left(m-\frac{M}{2}, n-\frac{N}{2}\right) & \cdots & v^{*}(m, n) & \cdots & v^{*}\left(m+\frac{M}{2}, n+\frac{N}{2}\right)
\end{array}\right) .
$$

3. Similarity Matching. Find the best-matching (winning) neuron $i(\mathbf{v})$ at time $t$, using the minimum complex Euclidean distance:

$$
i(\mathbf{v})=\underset{j}{\arg \min } \sum_{k=1}^{M x N} d\left(\mathbf{w}_{j, k}, \mathbf{v}_{k}\right),
$$

where $j=1,2, \ldots, L$. Scaling should be used in non-uniform color spaces. 
4. Updating. Adjust the synaptic weight vectors of all neurons, using the update formula

$$
\mathbf{w}_{j}(t+1)=\left\{\begin{array}{cl}
\mathbf{w}_{j}(t)+\eta(t)\left(\mathbf{v}(t)-\mathbf{w}_{j}(t)\right), & j \in \Lambda_{i(\mathbf{v})}(t) \\
\mathbf{w}_{j}(t), & \text { otherwise }
\end{array}\right.
$$

where $\eta(t)$ is the learning-rate parameter and $\Lambda_{i(\mathbf{v})}(t)$ is the neighborhood function centered around the winning neuron $i(\mathbf{v}) . \Lambda_{i(\mathbf{x}, \mathbf{v})}(t)$ are varied dynamically during learning for best results.

5. Continuation. Continue with step 2 until no noticeable changes in the feature map are observed.

Alternative, such distances as the weighted Minkowski metric, the Canberra distance and various similarity measures that are obtained by using different commonality and totality concepts [15] may be used instead of the Euclidean distance.

\subsection{Clustering}

The correlations of local color differences within the sliding window for color vectors represented by neuron $i$ are grouped into matrix $\mathbf{R}_{i}{ }_{i}$, which is defined as,

$$
\mathbf{R}_{i}^{\prime}=E\left[\Delta \mathbf{v}(m, n) \Delta \mathbf{v}^{\top}(m, n)\right]
$$

Assuming stationarity within the sliding window one may write,

$$
\mathbf{R}_{i}^{\prime}=\left(\begin{array}{ccccccccc}
\mathbf{R}_{i}(0,0) & \mathbf{R}_{i}(0,1) & \mathbf{R}_{i}(0,2) & \mathbf{R}_{i}(1,0) & \mathbf{R}_{i}(1,1) & \mathbf{R}_{i}(1,2) & \mathbf{R}_{i}(2,0) & \mathbf{R}_{i}(2,1) & \mathbf{R}_{i}(2,2) \\
\mathbf{R}_{i}(0,1) & \mathbf{R}_{i}(0,0) & \mathbf{R}_{i}(0,1) & \mathbf{R}_{i}(1,-1) & \mathbf{R}_{i}(1,0) & \mathbf{R}_{i}(1,1) & \mathbf{R}_{i}(2,-1) & \mathbf{R}_{i}(2,0) & \mathbf{R}_{i}(2,1) \\
\mathbf{R}_{i}(0,2) & \mathbf{R}_{i}(0,1) & \mathbf{R}_{i}(0,0) & \mathbf{R}_{i}(1,-2) & \mathbf{R}_{i}(1,-1) & \mathbf{R}_{i}(1,0) & \mathbf{R}_{i}(2,-2) & \mathbf{R}_{i}(2,-1) & \mathbf{R}_{i}(2,0) \\
\mathbf{R}_{i}(1,0) & \mathbf{R}_{i}(1,-1) & \mathbf{R}_{i}(1,-2) & \mathbf{R}_{i}(0,0) & \mathbf{R}_{i}(0,1) & \mathbf{R}_{i}(0,2) & \mathbf{R}_{i}(1,0) & \mathbf{R}_{i}(1,1) & \mathbf{R}_{i}(1,2) \\
\mathbf{R}_{i}(1,1) & \mathbf{R}_{i}(1,0) & \mathbf{R}_{i}(1,-1) & \mathbf{R}_{i}(0,1) & \mathbf{R}_{i}(0,0) & \mathbf{R}_{i}(0,1) & \mathbf{R}_{i}(1,-1) & \mathbf{R}_{i}(1,0) & \mathbf{R}_{i}(1,1) \\
\mathbf{R}_{i}(1,2) & \mathbf{R}_{i}(1,1) & \mathbf{R}_{i}(1,0) & \mathbf{R}_{i}(0,2) & \mathbf{R}_{i}(0,1) & \mathbf{R}_{i}(0,0) & \mathbf{R}_{i}(1,-2) & \mathbf{R}_{i}(1,-1) & \mathbf{R}_{i}(1,0) \\
\mathbf{R}_{i}(2,0) & \mathbf{R}_{i}(2,-1) & \mathbf{R}_{i}(2,-2) & \mathbf{R}_{i}(1,0) & \mathbf{R}_{i}(1,-1) & \mathbf{R}_{i}(1,-2) & \mathbf{R}_{i}(0,0) & \mathbf{R}_{i}(0,1) & \mathbf{R}_{i}(0,2) \\
\mathbf{R}_{i}(2,1) & \mathbf{R}_{i}(2,0) & \mathbf{R}_{i}(2,-1) & \mathbf{R}_{i}(1,1) & \mathbf{R}_{i}(1,0) & \mathbf{R}_{i}(1,-1) & \mathbf{R}_{i}(0,1) & \mathbf{R}_{i}(0,0) & \mathbf{R}_{i}(0,1) \\
\mathbf{R}_{i}(2,2) & \mathbf{R}_{i}(2,1) & \mathbf{R}_{i}(2,0) & \mathbf{R}_{i}(1,2) & \mathbf{R}_{i}(1,1) & \mathbf{R}_{i}(1,0) & \mathbf{R}_{i}(0,2) & \mathbf{R}_{i}(0,1) & \mathbf{R}_{i}(0,0)
\end{array}\right)
$$

which is a block symmetrical matrix and $\mathbf{R}_{i}(\Delta m, \Delta n)$ are $3 \times 3$ correlation submatrices for difference color vectors in $L^{*} u^{*} v^{*}$ color space $(\Delta m, \Delta n)$ pixels apart. A single linear neuron implementing a Hebbian-type adaptation rule for the training of its synaptic weights can evolve into a filter for the first principal component of the input distribution, $\mathbf{R}_{i} \mathbf{u}_{i}^{0}=\lambda_{i}^{0} \mathbf{u}_{i}^{0}$ ([16]). Several generalizations for multiple principal component analysis exist. Nevertheless one may apply the Householder transformation (see for example [17]) in order to obtain the eigenvectors and the eigenvalues for such a matrix by multiplying $\mathbf{R}_{i}{ }_{i}$ from the left and from the right by a sequence of matrices of the form $\left.\mathbf{P}_{k}=\operatorname{diag}\left\{1 \cdots \mathbf{A}_{k} \cdots 1\right\}-\mathbf{U}_{k}\left(\mathbf{H}_{k}^{-1}\right)_{3 \times 3} \mathbf{U}_{k}^{\prime \top}\right)$, where, 


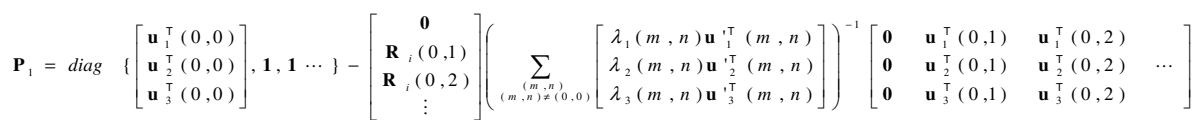

for $k=1$ with $\mathbf{u}_{l}^{\top}(m, n) \mathbf{R}_{i}(m, n) \mathbf{u}_{l}^{\prime}(m, n)=\operatorname{diag}\left\{\lambda_{l}(m, n), 0,0\right\}$ and likewise for other $k$.

\subsection{Segmentation}

Image segmentation consists of determining $K$ disjoint segments of an image, denoted as $I$, that are compact in image space, feature smooth boundaries and are homogeneous regarding color distribution within each region, i.e. a partition

$$
P(I)=\left\{R_{1}, R_{2}, R_{3}, \ldots, R_{K}\right\},
$$

where $R_{i} \cap R_{j}=\varnothing$ with $i, j \subset[1, K]$ and $i \neq j$. Segmentation algorithms seek to outline salient regions of an image. Often, their goal is to detect boundaries or interfaces between different regions. Such operators as the "Canny operator" for color edge detection and the Cumani operator are used [18]. Bayesian methods, level-set methods [19], active contour models [20], Gibbs models [21], watershed methods [22] and texture analysis methods [23] are widely used approaches. Segmentation algorithms are implemented as a first processing step in pattern classification, robot vision, medical image analysis, image coding and many other disciplines.

\section{Numerical Simulations}

\subsection{Original Images}

The medical images used to apply the proposed approach to image segmentation are selected from a histological database that has been developed in the University Hospital of the Democritus University of Thrace (DUTH), Greece, as well as standard medical images in free internet databases. The image in Fig. 1.a depicts a Bone Haversian System (UCLA Histology Collection). This is an example of compact bone. A Haversian system consists of concentric layers (lamellae) of bone surrounding a central Haversian canal in which blood vessels and nerves are located. The osteocytes are found within small lacunae. Fig. 1.c depicts a right and a left kidney (University Hospital, DUTH). There is intensive heterogeneity in the fixation of the radioisotope in the cortex of the right and left kidneys. Also, there are defective areas of radioisotope uptake in the upper and lower poles of both kidneys (particularly in the lower poles) as a scar. Figs. 1.b and 1.d illustrate the distributions of $L^{*} u^{*} v^{*}$ color vectors respectively. 


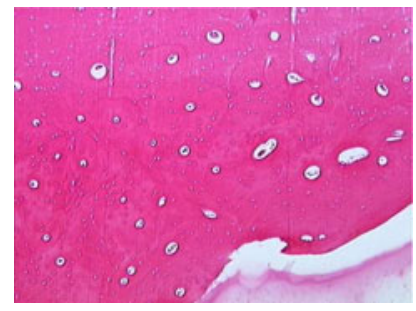

Fig. 1. a Bone - Haversian System (UCLA Histology Collection)

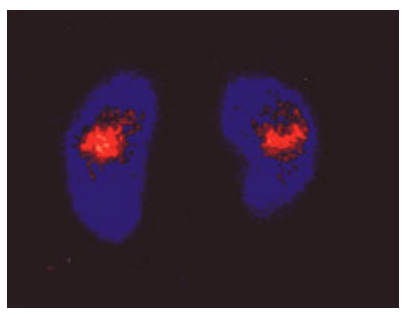

Fig. 1. c Right and left kidney (University Hospital, DUTH)

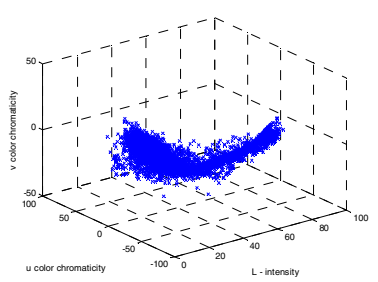

Fig. 1. b Distribution of vectors in $L^{*} u^{*} v^{*}$ color space

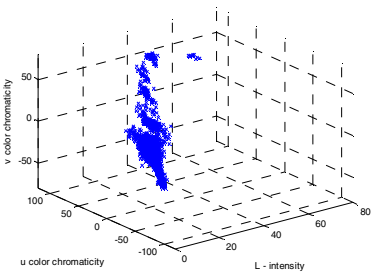

Fig. 1. d Distribution of vectors in $L^{*} u^{*} v^{*}$ color space

\subsection{Initialization and Training}

A 15 by 15 node SOFM initialized uniformly in a $3 \times 3 \times 3$ dimensional space is used to obtain representative points of the initial distribution. The training cycle consists of 100 epochs for both images and the learning rate parameter varies dynamically. The SOFMs for the average color vectors within the $3 \times 3$ sliding window after training are depicted in Figs. 2.a and 2.d. The corresponding color windows are illustrated in Figs. 2.b and 2.e. One may utilize the obtained values in order to construct a color reduced image, i.e. Color $\in\left\{\operatorname{Color}\left(\mathbf{w}_{1}\right) \cdots \operatorname{Color}\left(\mathbf{w}_{j}\right) \cdots \operatorname{Color}\left(\mathbf{w}_{L}\right)\right\}$.

The color reduced images for the two cases under consideration are given in Fig. 2.c and 2.f.
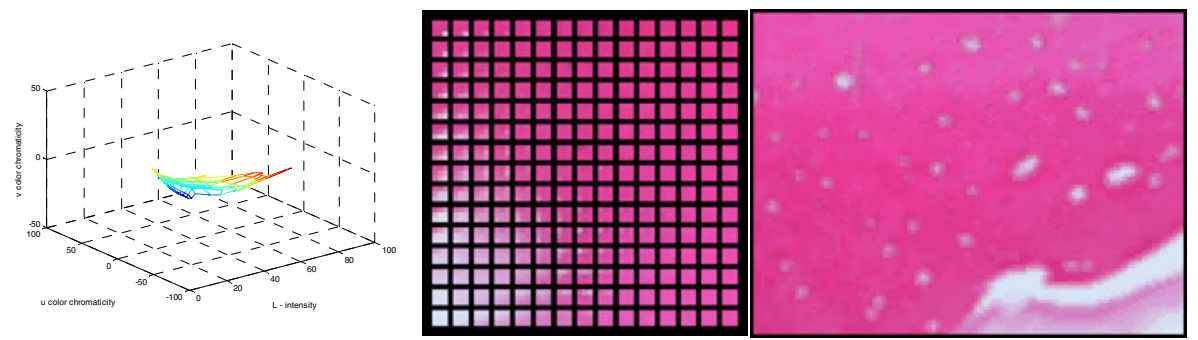

Fig. 2. a Distribution of average Fig. 2. b $15 \times 15$ SOFM color vectors in a sliding $3 \times 3$ after training window (Fig. 1.a)

Fig. 2. c Color reduced image 


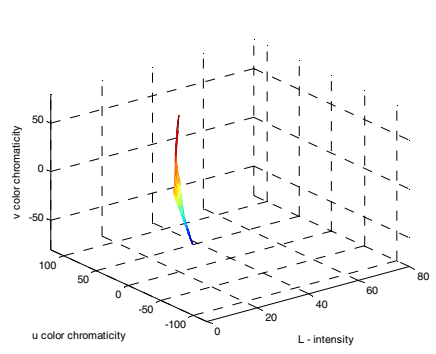

Fig. 2. d Distribution of average Fig. 2. e $15 \times 15$ SOFM color vectors in a sliding $3 \times 3$ after training window (Fig. 1.c)

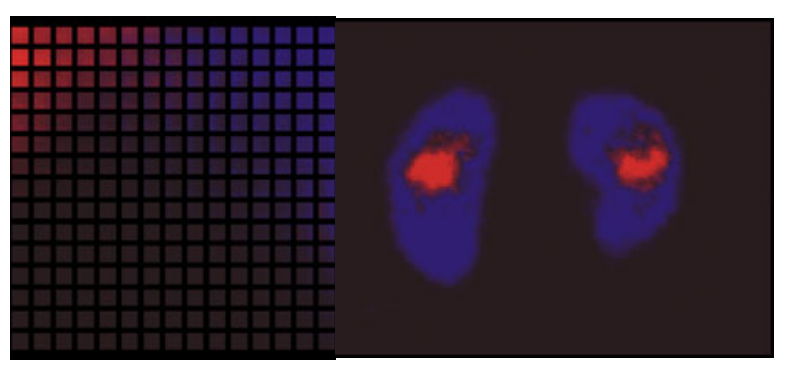

Fig. 2. f Color reduced image

\subsection{Clustering and Segmentation}

The local difference distribution of $\Delta \mathbf{v}=\left(\mathbf{w}_{j}-\mathbf{v}\right)$ for $i(\mathbf{v})=j$ is approximated by the eigenvectors corresponding to its largest eigenvalues. Standard SVD algorithms indicate that pdfs featuring a dimensionality of eight (8) eigenvectors approximate at least $90 \%$ of the power spectrum of the local distributions (see Figs. 3.a and 3.b for both images). Nevertheless for the sake of simplicity only the three (3) largest eigenvectors - denoted as $\mathbf{w}_{j, 1}^{e}, \mathbf{w}_{j, 2}^{e}$ and $\mathbf{w}_{j, 3}^{e}$ - are used in order to measure the similarity between local distributions. The similarity between local pdfs according to the Mahalanobis distance [24] is estimated as,

$$
\begin{aligned}
\frac{1}{2}\left(\mathbf{w}_{j}-\mathbf{w}_{k}\right)^{\top}\left[\mathbf{w}_{j, 1}^{e} \frac{1}{\lambda_{1}}\left(\mathbf{w}_{j, 1}^{e}\right)^{\top}+\mathbf{w}_{j, 2}^{e} \frac{1}{\lambda_{2}}\left(\mathbf{w}_{j, 2}^{e}\right)^{\top}+\mathbf{w}_{j, 3}^{e} \frac{1}{\lambda_{3}}\left(\mathbf{w}_{j, 3}^{e}\right)^{\top}+\right. \\
\left.\mathbf{w}_{k, 1}^{e} \frac{1}{\lambda_{1}}\left(\mathbf{w}_{k, 1}^{e}\right)^{\top}+\mathbf{w}_{k, 2}^{e} \frac{1}{\lambda_{2}}\left(\mathbf{w}_{k, 2}^{e}\right)^{\top}+\mathbf{w}_{k, 3}^{e} \frac{1}{\lambda_{3}}\left(\mathbf{w}_{k, 3}^{e}\right)^{\top}\right]\left(\mathbf{w}_{j}-\mathbf{w}_{k}\right)<t h
\end{aligned}
$$

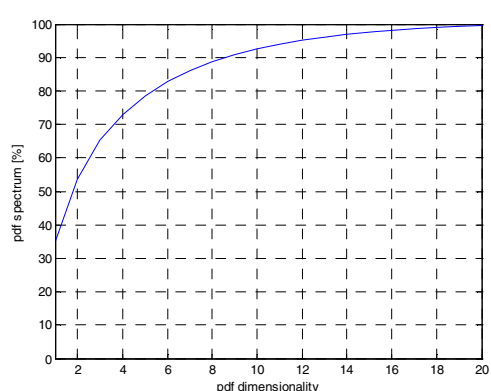

Fig. 3. a Spectrum of eigenvalues vs. number of eigenvectors (Bone - Haversian System)

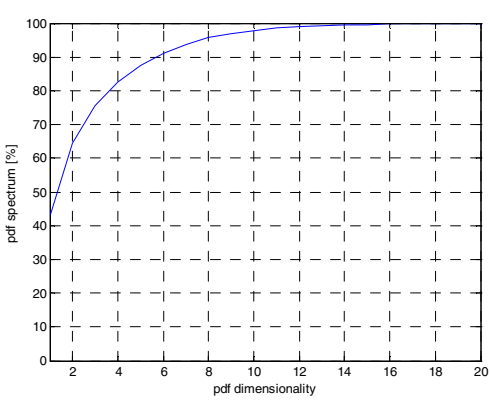

Fig. 3. b Spectrum of eigenvalues vs. number of eigenvectors (Right and left kidney) 

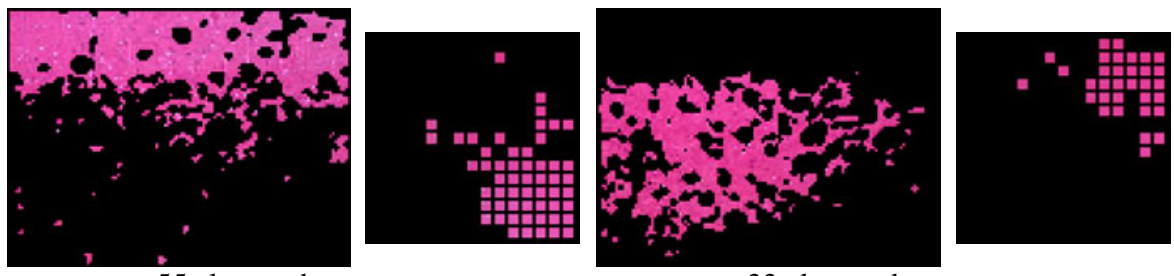

55 clustered neurons
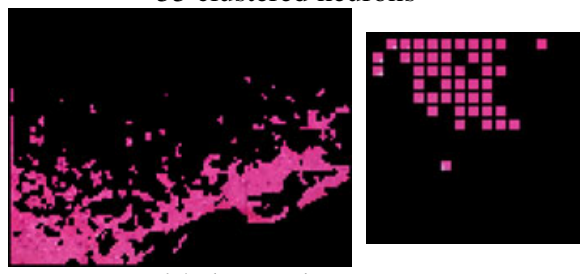

45 clustered neurons
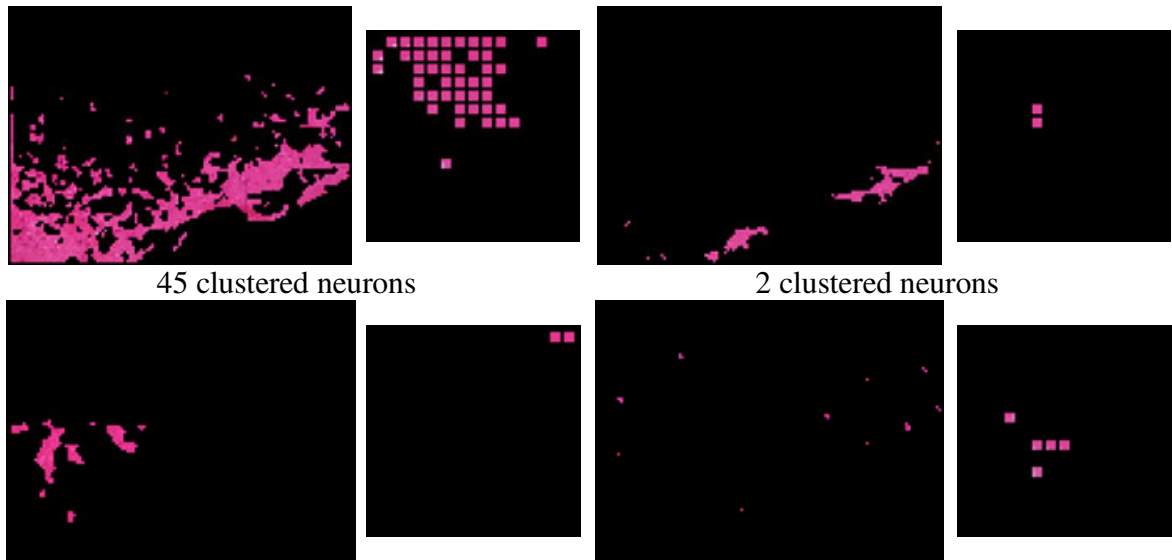

2 clustered neurons

2 clustered neurons

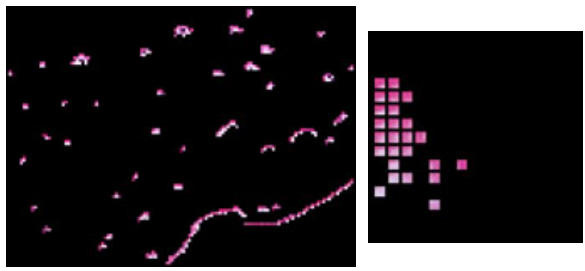

25 clustered neurons
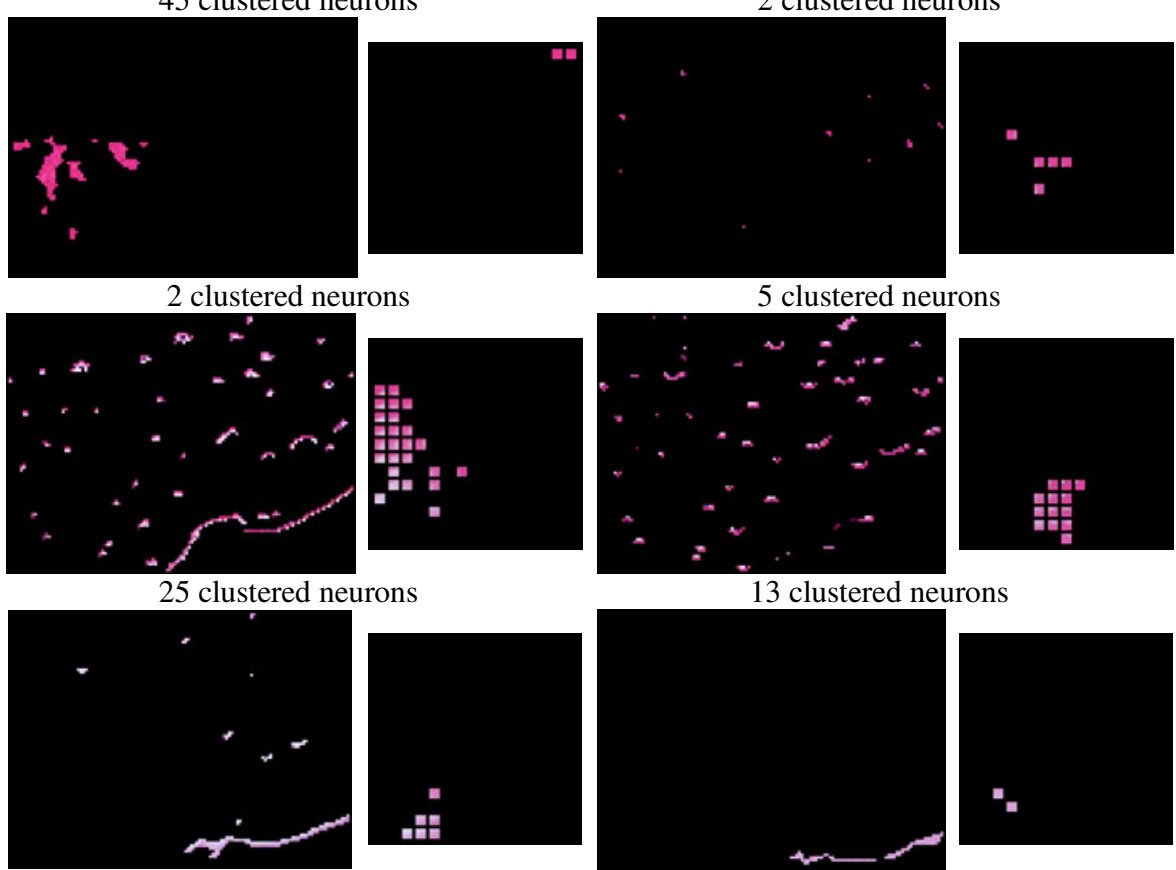

5 clustered neurons

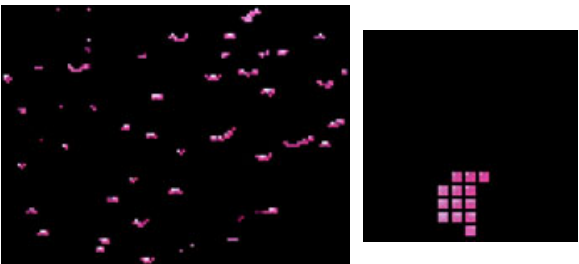

13 clustered neurons

6 clustered neurons
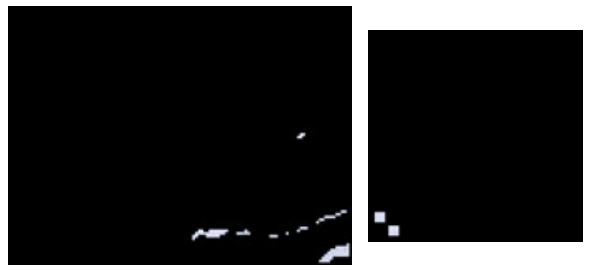

2 clustered neurons

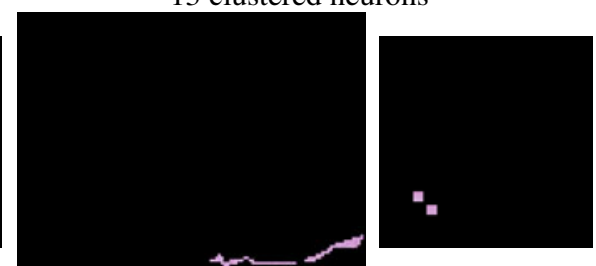

2 clustered neurons

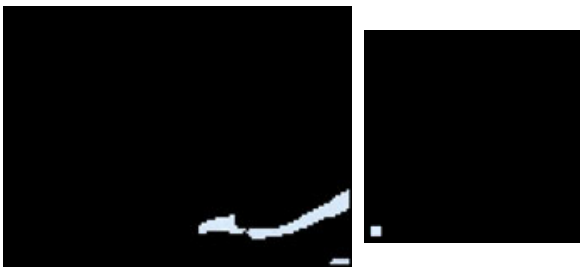

1 neuron

Fig. 4. Image segments (left images) attributed to clusters of neurons of the SOFM after training (depicted on the right images) for the Bone - Haversian System (Fig. 1a) 


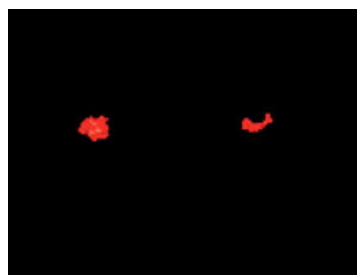

1 neuron

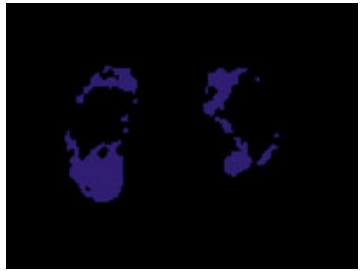

2 clustered neurons
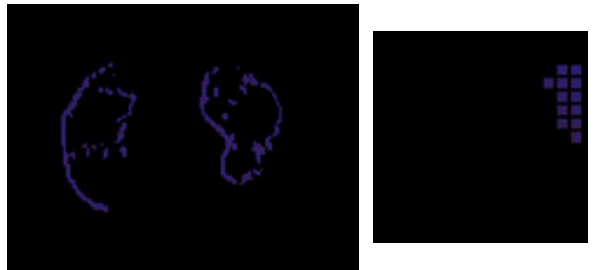

12 clustered neurons

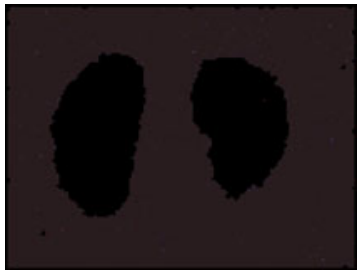

89 clustered neurons
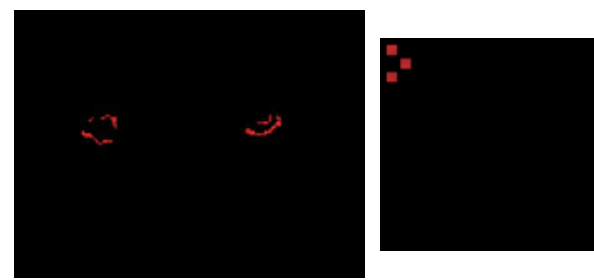

3 clustered neurons
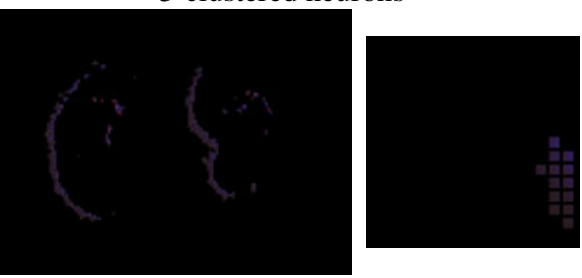

13 clustered neurons
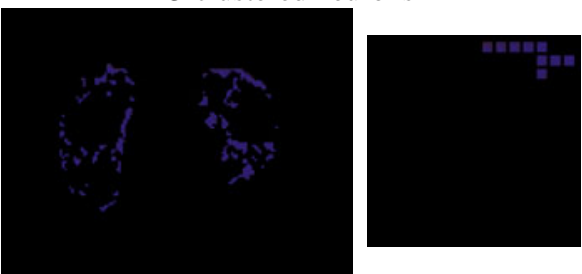

9 clustered neurons

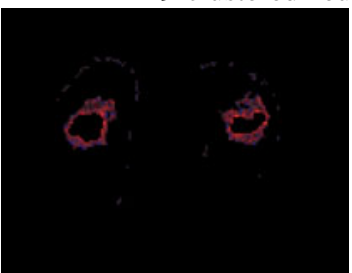

40 clustered neurons

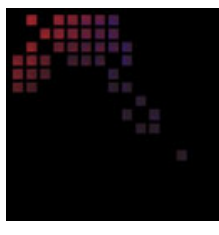

Fig. 5. Image segments (left images) attributed to clusters of neurons of the SOFM after training (depicted on the right images) for the right and left kidney in Fig. 1b

One may use alternative measures and clustering schemes as well like - for example - the partition dissimilarity functions presented in [25].

\section{Discussion}

Groups of segments for the two images as well as the corresponding clustered neurons are presented in Figs. 4 and 5. Spatial nearest-neighbor assignment of pixels to regions enhances their compactness. Both color and structural details characterize the obtained segments. Regions exhibiting smooth red, blue and pink color without variations are prominent groups of segments in the processed images. Regions with textural variations of red and blue color as well as directional variations of pink and blue are grouped separately. Background clusters most of the neurons in Fig. 1.c and 
forms a segment of the image by itself. Different segments correspond to different tissues and underlying biological structures. The number of eigenvectors chosen to represent local distributions of color vectors affects both the accuracy of the segmentation algorithm and its execution time. The size of the sliding window is also a crucial factor that affects the execution time of the proposed algorithm.

\section{References}

1. Ramanath, R., Snyder, W.E., Yoo, Y., Drew, M.S.: Color Image Processing Pipeline. IEEE Signal Processing Mag. 22(1), 34-43 (2005)

2. Cucchiara, R., Grana, C.: Color Analysis, Segmentation and Retrieval in Medical Imaging

3. Koschan, A., Abidi, M.: Detection and Classification of Edges in Color Images. IEEE Signal Processing Mag. 22(1), 64-73 (2005)

4. Karvelis, P.S., Fotiadis, D.I.: Enhancement of Multispectral Chromosome Image Classification Using Vector Median Filtering. In: Proc. 15th Int. Conference on Conceptual Structures (ICCS 2007), Sheffield, England (2007)

5. Mosaliganti, K., Janoos, F., Irfanoglu, O., Ridgway, R., Machiraju, R., Huang, K., Saltz, J., Leone, G., Ostrowski, M.: Tensor Classification of N-Point Correlation Function Features For Histology Tissue Segmentation. Medical Image Analysis 13, 156-166 (2009)

6. Robertson, A.R.: The CIE 1976 Color-difference Formulae. Color Research and Applications 2, 7-11 (1977)

7. Tominaga, S.: Color Classification of Natural Color Images. Color Research and Applications 17, 230-239 (1992)

8. Pitas, I., Venetsanopoulos, A.N.: Nonlinear Digital Filters, Principles and Applications. Kluwer, Norwell (1990)

9. Lukac, R., Smolka, B., Martin, K., Plataniotis, K.N., Venetsanopoulos, A.N.: Vector Filtering for Color Imaging. IEEE Signal Processing Mag. 22(1), 74-86 (2005)

10. Astola, J., Haavisto, P., Neuvo, Y.: Vector Median Filters. Proc. IEEE 78(4), 678-689 (1990)

11. Kohonen, T.: Self-Organizing Maps. Series in Information Sciences. Springer, Berlin (1995)

12. Stephanakis, I., Anastassopoulos, G., Iliadis, L.: Color Segmentation Using SelfOrganizing Feature Maps (SOFMs) Defined Upon Color and Spatial Image Space. In: Proc. 20th International Conference on Artificial Neural Networks, Thessaloniki, Greece (2010)

13. Yin, H.: ViSOM - A Novel Method for Multivariate Data Projection and Structure Visualization. IEEE Trans. Neural Networks 13(1), 237-243 (2002)

14. Jiang, X.: Linear Subspace Learning-Based Dimensionality Reduction. IEEE Signal Processing Mag. 28(2), 16-26 (2011)

15. Plataniotis, K.N., Androutsos, D., Venetsanopoulos, A.N.: Adaptive Fuzzy Systems for Multichannel Signal Processing. Proc. IEEE 87(9), 1601-1622 (1999)

16. Oja, E.: A Simplified Neuron Model as a Principal Component Analyzer. Journal of Mathematical Biology 15, 267-273 (1982)

17. Golub, G.H., Van Loan, C.F.: Matrix Computations, 2nd edn. Johns Hopkins University Press, Baltimore (1989)

18. Koschan, A., Abidi, M.: Detection and Classification of Edges in Color Images. IEEE Signal Processing Mag. 22(1), 64-73 (2005) 
19. Malladi, R., Sethian, J.A., Vermuri, B.C.: Shape Modeling With Front Propagation: A Level set Approach. IEEE Trans. on Pattern Analysis and Machine Intelligence 17(2), 158-174 (1995)

20. Caselles, V., Kimmel, R., Sapiro, G.: Geodesic Active Contours. International Journal on Computer Vision 22(1), 61-97 (1997)

21. Chen, T., Metaxas, D.: A Hybrid Framework for 3D Medical Image Segmentation. Medical Image Analysis 9, 547-565 (2005)

22. Vincent, L., Soille, P.: Watersheds in Digital Spaces: An Efficient Algorithm Based on Immersion Simulations. IEEE Trans. Pattern Analysis and Machine Intelligence 13(6), 583-598 (1991)

23. Haralik, R., Shanmugam, K., Dinstein, I.: Textural Features for Image Classification. IEEE Trans. on Systems, Man and Cybernetics, 610-621 (1973)

24. McLachlan, G.J.: Discriminant Analysis and Statistical Pattern Recognition, p. 12. Wiley Interscience, Hoboken (1992)

25. Logeswari, T., Karnan, M.: An Improved Implementation of Brain Tumor Detection Using Segmentation Based on Hierarchical Self Organizing Maps. International Journal of Computer Theory and Engineering 2(4), 591-595 (2010) 